\title{
Database Modeling and Visualization Simulation technology Based on Java3D

\author{
Hongxia Liu
}

Department of Electronic Information Engineering, Handan Polytechnic College, Handan, 056001, China

liuhonxia@163.com

Keywords: Java3D; Database; Visualization; Simulation; Color rendering; Nephogram

\begin{abstract}
Computer simulation technology has been applied to many industries and fields, due to its intuitive, convenient and interactive visualization. Java3D programming technology is easy to be operated, and its cross platform performance is good, and it is easy to develop web-based applications, so it is the common database visual modeling and simulation technologies. In this paper, the data field visualization algorithm is established using Java3D technology, and the color rendering has been done for plane and three-dimensional sphere, which got a good visualization effect. 3D database grid model has been designed, and three-dimensional database images have been got through the color line rendering, which provides the theory reference for the research on visualization method for the database.
\end{abstract}

\section{Introduction}

The role of Java 3D API is a complex interface database of three-dimensional graphics and sound rendering system, programmers only need finished the work of creation and control of high-rise three dimensional geometric object, which can complete visualization operation of the database. These geometric objects is in a virtual world, which is rendered objects. The method can be built from micro to macro atomic virtual scene of the universe, and the whole rendering details can through the system to achieve automatic control, the Java 3D application development process can be broadly divided into the following steps.

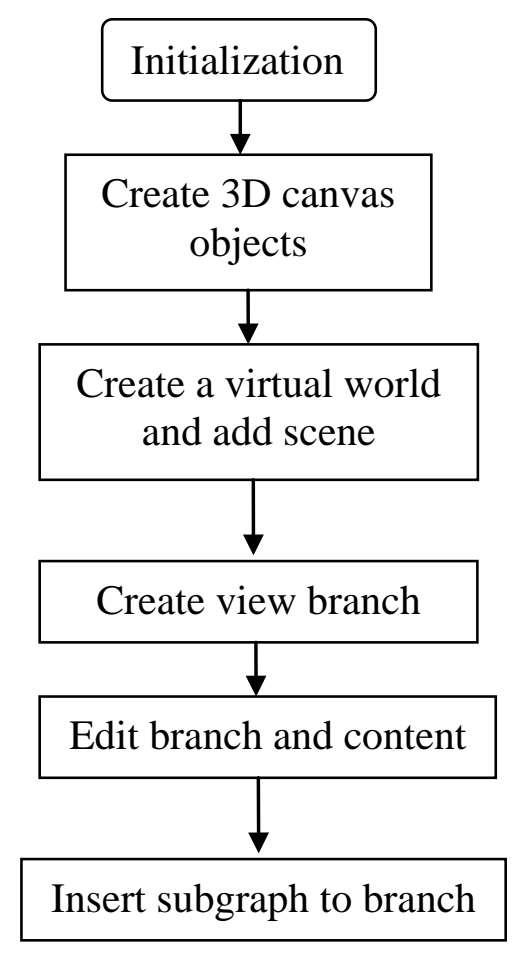

Fig.1 Java 3D application development flow chart 
As shown in Figure 1 represents the Java3D application development process flow chart, Java 3D creates an instance from then to the realization of visual programming these examples in the scene graph data structure. Java3D visualization operations can be implemented on a large scale database, can be directly drawing and rendering of the measured database. The researchers could not learn professional knowledge and other aspects of the use of the method of data processing, including processing includes curves, plane, sphere, spectrum etc..

\section{Optimization model and Algorithm design of database Visualization Modeling}

In this paper, the mathematical base of database visual modeling and optimization is spatial statistics analysis of data. Assuming that the database data space matrix is $S$, and the matrix is symmetric, then the database space of n-dimensional proximity relation can also use matrix expressed, the expression as shown in formula (1).

$$
S=\left[\begin{array}{cccc}
S_{11} & S_{12} & \cdots & S_{1 n} \\
S_{21} & S_{22} & \cdots & S_{2 n} \\
\cdots & \cdots & \cdots & \cdots \\
S_{n 1} & S_{n 2} & \cdots & S_{n n}
\end{array}\right]
$$

(1)In formula (1), $s_{i j}$ represents the adjacent relations of the data point $i$ and data point $j$ network space, if the $x_{i}$ is reference value of data $i$, then the similarity between data points can be represented as shown in formula (2).

$$
\begin{gathered}
I=\frac{n \sum_{i=1}^{n} \sum_{j=1}^{n} q_{i j}\left(x_{i}-\bar{x}\right)\left(x_{j}-\bar{x}\right)}{\sum_{i=1}^{n} \sum_{j=1}^{n} q_{i j} \sum_{i=1}^{n}\left(x_{i}-\bar{x}\right)^{2}} \\
=\frac{\sum_{i=1}^{n} \sum_{j \neq i}^{n} q_{i j}\left(x_{i}-\bar{x}\right)\left(x_{j}-\bar{x}\right)}{S^{2} \sum_{i=1}^{n} \sum_{j \neq i}^{n} q_{i j}}
\end{gathered}
$$

(2)In formula (2), $S$ is data space distance variance, it's formula form can be written as formula (3):

$S^{2}=\frac{1}{n} \sum_{i}\left(x_{i}-\bar{x}\right)^{2}$

(3)In formula (3), $\bar{x}$ is average distance, it's formula form can be written as formula (4):

$\bar{x}=\frac{1}{n} \sum_{i=1}^{n} x_{i}$

(4)In this paper, the optimization parameter $Y$ has been introduced to the data clustering, which can realize the optimization function of database, the expression as shown in formula (5).

$$
Y=\frac{(n-1) \sum_{i=1}^{n} \sum_{j=1}^{n} w_{i j}\left(x_{i}-x_{j}\right)^{2}}{2 \sum_{i=1}^{n} \sum_{j=1}^{n} w_{i j} \sum_{i=1}^{n}\left(x_{i}-\bar{x}\right)^{2}}
$$

(5)Java3D can realize the database visual modeling and simulation and rendering using database optimization algorithm, the invoked method is setCoordinate and setColor, where in the pseudo code of the main algorithm is as follows:

line=LineStripArray (line.COORDINATES | line.COLOR_3, stripvertexCount) 
line.setCoordinate(0,coordinate_V0)line.setColor(0,color_0)line.setCoordiante(1,coordinate_V1) line.setColor(1,color_1)line.setCoordiante(2,coordinate_V1)line.setColor(2,color_1)line.setCoordia nte(3,coordinate_V2)line.setColor(3,color_2)......

\section{Study on visual modeling and simulation rendering calculation of Java3D database}

In order to verify the second section design database visualization optimization mathematical model and the validity and reliability of the algorithm, this paper uses Java3D technology for 2D images were rendered, in $2 \mathrm{~d}$ rendering process, the four vertex select image set the reference point coordinate and color, finally complete the grid area rendering.

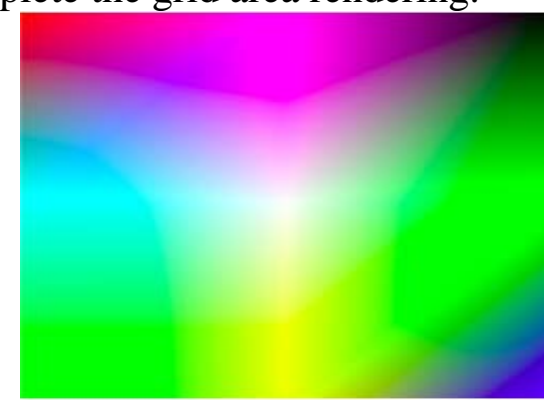

Fig.2 Two dimensional rendering results

In Figure 2, it shows a two-dimensional visualization database by using Java3D technology rendering results, very good has completed filled by rendering the color value. In order to verify the algorithm, this paper selects the three-dimensional energetic electron flux spatial database spherical display, the result shown in figure 3.

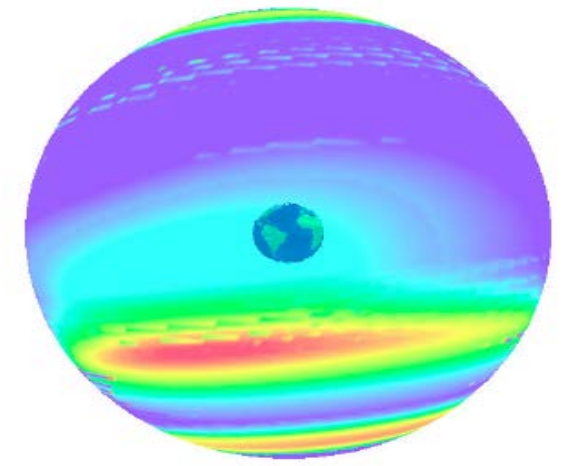

Fig. 3 The visualization results of energetic electron flux 3D spatial database

As shown in Figure 3, the coordinate and color of these reference points have been set by using magnetic field of two dimensional plane rectangle method and Set Coordinate Index method in the paper, the sphere is partitioned into 180*359 small rectangular, which can ensure that such a vertex position relatively close, so the smooth visual sphere has been formed.

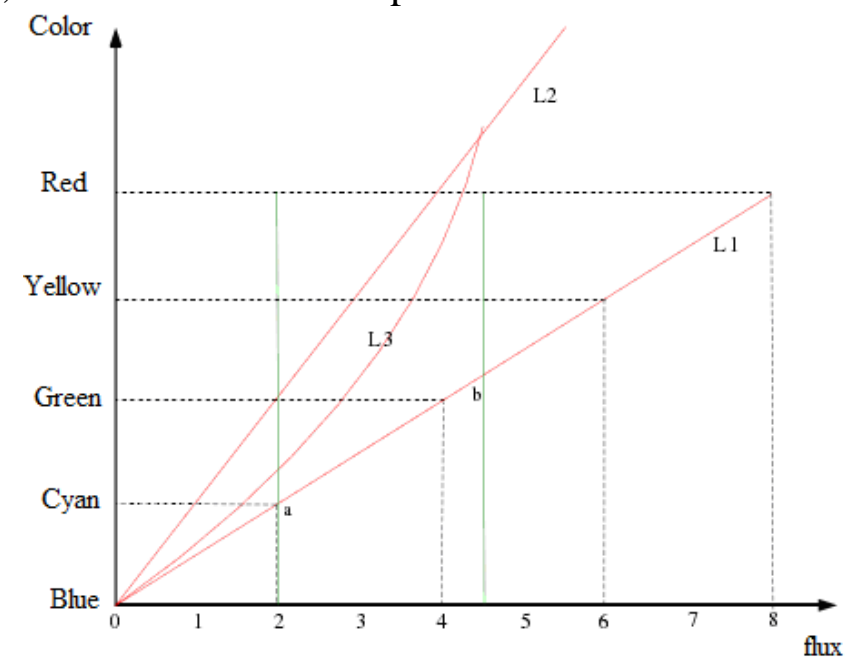

Fig. 4 Energetic particle flux data analysis chart 
Figure 4 shows the curve analysis of energetic particle flux data, the data flux in this study were divided into three groups, in order to realize visualization of the database shows need to establish visual grid model using Java3D technology, the visualization grid as shown in figure 5.

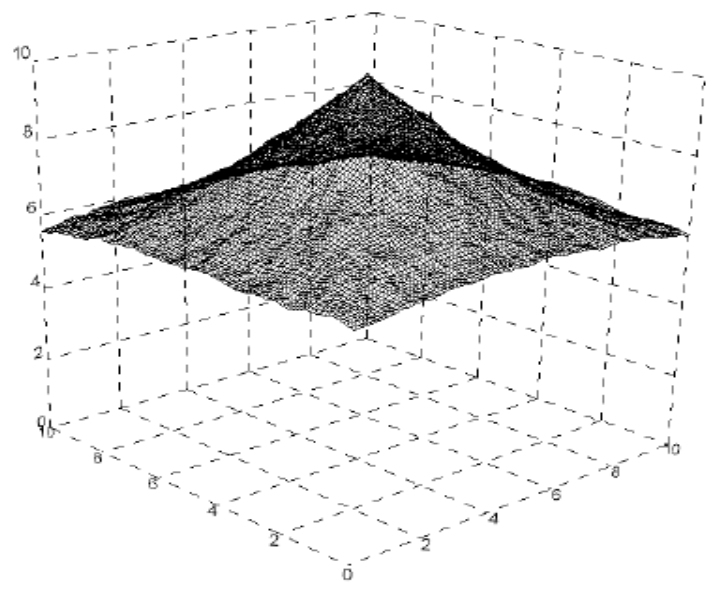

Fig.5 3D database visual modeling

As shown in Figure 5 represented by visual data model is established by Java3D, the model grid using elevation data of linear fractal method to generate, through iterative calculation can be carried out after the color rendering of model, the visualization results as shown in figure 6 .

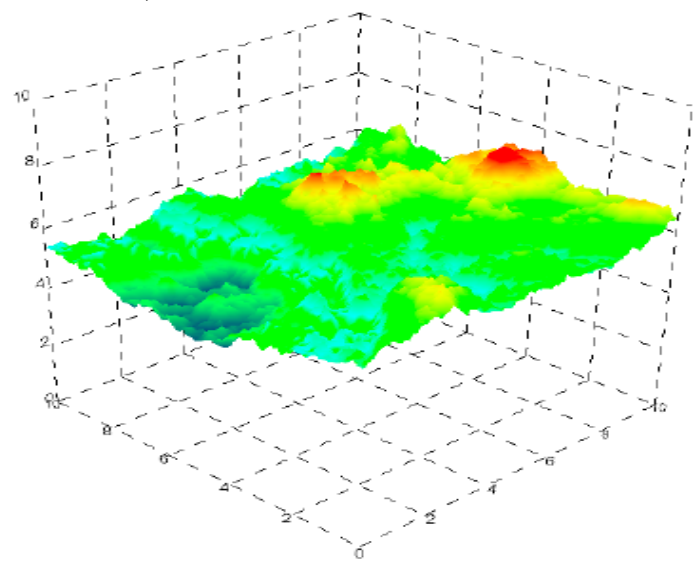

Fig.6 Visual rendering results

The use of Java3D after 3D visualization rendering of color obtained results indicated in Figure 6 , through 2000 iterations, realize color line simulation. Thus it can be seen that the function of visual three-dimensional simulation realization of 3D spatial data field based on Java3D, has the broad application prospect.

\section{Conclusion}

(1) On the basis of spatial statistical analysis mathematical principle to establish the database of visual optimization model and algorithm, and the design of pseudo code visualization rendering using Java3D programming technology.

(2) The visual space of some physical factors near field as an example, introduces in detail how to realize the visualization of large scale data field with Java3D, which verifies the validity and reliability of the visualization method of Java3D database.

(3) The plane and three-dimensional color rendering have been got, taking the modeling and simulation of 3D terrain field in the field of geographic information visualization as an example, which provide technical reference for visualization research database.

\section{References}

[1] Li Qian, Ji Xiaomin, Wang Mingliang. Virtual human simulation method based on Java3D. The application of computer, Vol.30 (11), (2010), p.3084-3086. 
[2] Kang arts, Zhang Dinghua, Yu Suihuai. Study on the key method of converting the color analyzer color. Computer engineering and applications, Vol.46 (1), (2010), p.51-53.

[3] Jiang Yu, Durbin, Lu Jun. Algorithm of drawing isoline based on Delaunay triangle net. Computer Application Research, Vol.27 (1), (2010),p.101-103.

[4] Li Na. Data mining software products are reviewed in this paper. The popular science and technology, Vol.01 (2010), p.22-24.

[5] Deng Qianni, Chen Quan. Cloud computing and its key technology. Application and development of high performance computing. Vol.26 (1), (2009), p.2-6.

[6] Li Xiaopeng. Electronic purse system design and security research based on NFC technology. Beijing: Beijing University of Posts and Telecommunications, (2007):p.25-33.

[7] Xia Wendong, Lin Kai. 3G NFC smart card system fused. Computer Engineering,Vol.37, (2011), p.230-239.

[8] Deng Haisheng, Li Junhuai. Mobile computing mode based on reflective middleware. Computer Engineering.Vol.38 (20), (2012), p.278-281.

[9] Zheng Di, Wang Jun. A member for supporting context aware adaptation model research on. Computer Engineering, Vol.38 (2), (2012), p.39-41.

[10] Wang Huajin. Survey on the visualization of data mining. Technology Plaza, Vol.01, (2009): p.235-237. 